\title{
A Study of Cho-Kwon-Srivastava Operator with Applications to Generalized Hypergeometric Functions
}

\author{
F. Ghanim ${ }^{1}$ and M. Darus ${ }^{2}$ \\ ${ }^{1}$ Department of Mathematics, College of Sciences, University of Sharjah, Sharjah, UAE \\ ${ }^{2}$ School of Mathematical Sciences, Faculty of Science and Technology, Universiti Kebangsaan Malaysia, \\ 43600 Bangi, Selangor, Malaysia \\ Correspondence should be addressed to M. Darus; maslina@ukm.edu.my
}

Received 16 May 2014; Accepted 21 June 2014; Published 9 July 2014

Academic Editor: Hari M. Srivastava

Copyright (C) 2014 F. Ghanim and M. Darus. This is an open access article distributed under the Creative Commons Attribution License, which permits unrestricted use, distribution, and reproduction in any medium, provided the original work is properly cited.

\begin{abstract}
We introduce a new class of meromorphically analytic functions, which is defined by means of a Hadamard product (or convolution) involving some suitably normalized meromorphically functions related to Cho-Kwon-Srivastava operator. A characterization property giving the coefficient bounds is obtained for this class of functions. The other related properties, which are investigated in this paper, include distortion and the radii of starlikeness and convexity. We also consider several applications of our main results to generalized hypergeometric functions.
\end{abstract}

\section{Introduction}

A meromorphic function is a single-valued function that is analytic in all but possibly a discrete subset of its domain, and at those singularities it must go to infinity like a polynomial (i.e., these exceptional points must be poles and not essential singularities). A simpler definition states that a meromorphic function $f(z)$ is a function of the form

$$
f(z)=\frac{g(z)}{h(z)},
$$

where $g(z)$ and $h(z)$ are entire functions with $h(z) \neq 0$ (see [1, page 64]). A meromorphic function therefore may only have finite-order, isolated poles and zeros and no essential singularities in its domain. An equivalent definition of a meromorphic function is a complex analytic map to the Riemann sphere. For example, the gamma function is meromorphic in the whole complex plane $\mathbb{C}$.

In the present paper, we initiate the study of functions which are meromorphic in the punctured disk $U^{*}=\{z: 0<$ $|z|<1\}$ with a Laurent expansion about the origin; see [2].
Let $A$ be the class of analytic functions $h(z)$ with $h(0)=1$, which are convex and univalent in the open unit disk $U=$ $U^{*} \cup\{0\}$ and for which

$$
\Re\{h(z)\}>0, \quad\left(z \in U^{*}\right) .
$$

For functions $f$ and $g$ analytic in $U$, we say that $f$ is subordinate to $g$ and write

$$
f \prec g \text { in } U \text { or } f(z) \prec g(z), \quad\left(z \in U^{*}\right)
$$

if there exists an analytic function $w(z)$ in $U$ such that

$$
|w(z)| \leq|z|, \quad f(z)=g(w(z)), \quad\left(z \in U^{*}\right) .
$$

Furthermore, if the function $g$ is univalent in $U$, then

$$
\begin{gathered}
f(z) \prec g(z) \Longleftrightarrow f(0)=g(0), \\
f(U) \subseteq g(U), \quad\left(z \in U^{*}\right) .
\end{gathered}
$$

This paper is divided into two sections; the first introduces a new class of meromorphically analytic functions, which is defined by means of a Hadamard product (or 
convolution) involving linear operator. The second section highlights some applications of the main results involving generalized hypergeometric functions.

\section{Preliminaries}

Let $\Sigma$ denote the class of meromorphic functions $f(z)$ normalized by

$$
f(z)=\frac{1}{z}+\sum_{n=1}^{\infty} a_{n} z^{n}
$$

which are analytic in the punctured unit disk $U^{*}=\{z: 0<$ $|z|<1\}$. For $0 \leq \beta$, we denote by $S^{*}(\beta)$ and $k(\beta)$ the subclasses of $\Sigma$ consisting of all meromorphic functions which are, respectively, starlike of order $\beta$ and convex of order $\beta$ in $U$.

For functions $f_{j}(z)(j=1 ; 2)$ defined by

$$
f_{j}(z)=\frac{1}{z}+\sum_{n=1}^{\infty} a_{n, j} z^{n}
$$

we denote the Hadamard product (or convolution) of $f_{1}(z)$ and $f_{2}(z)$ by

$$
\left(f_{1} * f_{2}\right)=\frac{1}{z}+\sum_{n=1}^{\infty} a_{n, 1} a_{n, 2} z^{n}
$$

Cho et al. [3] and Ghanim and Darus [4] studied the following function:

$$
q_{\lambda, \mu}(z)=\frac{1}{z}+\sum_{n=1}^{\infty}\left(\frac{\lambda}{n+1+\lambda}\right)^{\mu} z^{n}, \quad(\lambda>0, \mu \geq 0) .
$$

Corresponding to the function $q_{\lambda, \mu}(z)$ and using the Hadamard product for $f(z) \in \Sigma$, we define a new linear operator $L(\lambda, \mu)$ on $\Sigma$ by

$$
\begin{aligned}
L_{\lambda, \mu} f(z) & =\left(f(z) * q_{\lambda, \mu}(z)\right) \\
& =\frac{1}{z}+\sum_{n=1}^{\infty}\left(\frac{\lambda}{n+\lambda+1}\right)^{\mu}\left|a_{n}\right| z^{n} .
\end{aligned}
$$

The Hadamard product or convolution of the functions $f$ given by (10) with the functions $L_{t, a} g$ and $L_{t, a} h$ given, respectively, by

$$
\begin{array}{r}
L_{\lambda, \mu} g(z)=\frac{1}{z}+\sum_{n=1}^{\infty}\left(\frac{\lambda}{n+\lambda+1}\right)^{\mu}\left|b_{n}\right| z^{n}, \\
\left(z \in U^{*}, g(z) \in \Sigma\right), \\
L_{\lambda, \mu} h(z)=\frac{1}{z}+\sum_{n=1}^{\infty}\left(\frac{\lambda}{n+\lambda+1}\right)^{\mu}\left|c_{n}\right| z^{n}, \\
\left(z \in U^{*}, h(z) \in \Sigma\right),
\end{array}
$$

can be expressed as follows:

$$
\begin{array}{r}
L_{\lambda, \mu}(f * g)(z)=\frac{1}{z}+\sum_{n=1}^{\infty}\left(\frac{\lambda}{n+\lambda+1}\right)^{\mu}\left|a_{n} b_{n}\right| z^{n}, \\
\left(z \in U^{*}\right), \\
L_{\lambda, \mu}(f * h)(z)=\frac{1}{z}+\sum_{n=1}^{\infty}\left(\frac{\lambda}{n+\lambda+1}\right)^{\mu}\left|a_{n} c_{n}\right| z^{n}, \\
\left(z \in U^{*}\right) .
\end{array}
$$

By applying the subordination definition, we introduce here a new class $\Sigma_{\lambda}^{\mu}(\rho, A, B)$ of meromorphic functions, which is defined as follows.

Definition 1. A function $f \in \Sigma$ of the form (6) is said to be in the class $\Sigma_{\lambda}^{\mu}(\rho, A, B)$ if it satisfies the following subordination property:

$$
\rho \frac{L_{\lambda, \mu}(f * g)(z)}{L_{\lambda, \mu}(f * h)(z)}<\rho-\frac{(A-B) z}{1+B z}, \quad\left(z \in U^{*}\right),
$$

where $-1 \leq B<A \leq 1, \rho>0$, with condition $0 \leq\left|c_{n}\right| \leq\left|b_{n}\right|$ and $L(\lambda, \mu)(f * h)(z) \neq 0$.

As for the second result of this paper on applications involving generalized hypergeometric functions, we need to utilize the well-known Gaussian hypergeometric function. One denotes $\phi(\alpha, \beta ; z)$ the class of the function given by

$$
\tilde{\phi}(\alpha, \beta ; z)=\frac{1}{z}+\sum_{n=0}^{\infty} \frac{(\alpha)_{n+1}}{(\beta)_{n+1}} z^{n},
$$

for $\beta \neq 0,-1,-2, \ldots$, and $\alpha \in \mathbb{C} \backslash\{0\}$, where $(\lambda) n=\lambda(\lambda+1)_{n+1}$ is the Pochhammer symbol. We note that

$$
\widetilde{\phi}(\alpha, \beta ; z)=\frac{1}{z}{ }_{2} F_{1}(1, \alpha, \beta ; z),
$$

where

$$
{ }_{2} F_{1}(b, \alpha, \beta ; z)=\sum_{n=0}^{\infty} \frac{(b)_{n}(\alpha)_{n}}{(\beta)_{n}} \frac{z^{n}}{n !}
$$

is the well-known Gaussian hypergeometric function.

Corresponding to the functions $\widetilde{\phi}(\alpha, \beta ; z)$ and $q_{\lambda, \mu}(z)$ given in (9) and using the Hadamard product for $f(z) \in \Sigma$, we define a new linear operator $L(\alpha, \beta, \lambda, \mu)$ on $\Sigma$ by

$$
\begin{aligned}
L(\alpha, \beta, \lambda, \mu) f(z) & =\left(f(z) * \phi(\alpha, \beta ; z) * q_{\lambda, \mu}(z)\right) \\
& =\frac{1}{z}+\sum_{n=1}^{\infty} \frac{(\alpha)_{n+1}}{(\beta)_{n+1}}\left(\frac{\lambda}{n+\lambda+1}\right)^{\mu}\left|a_{n}\right| z^{n} .
\end{aligned}
$$

The meromorphic functions with the generalized hypergeometric functions were considered recently by Cho and Kim [5], Dziok and Srivastava [6,7], Ghanim [8], Ghanim et al. $[9,10]$, and Liu and Srivastava $[11,12]$. 
Now, it follows from (17) that

$$
\begin{aligned}
z(L(\alpha, \beta, \lambda, \mu) f(z))^{\prime}= & \alpha L(\alpha+1, \beta, \lambda, \mu) f(z) \\
& -(\alpha+1) L(\alpha, \beta, \lambda, \mu) f(z) .
\end{aligned}
$$

The subordination relation (13) in conjunction with (17) takes the following form:

$$
\begin{array}{r}
\rho \frac{L(\alpha+1, \beta, \lambda, \mu) f(z)}{L(\alpha, \beta, \lambda, \mu) f(z)} \prec \rho-\frac{(A-B) z}{1+B z}, \\
(0 \leq B<A \leq 1, \rho>0) .
\end{array}
$$

Definition 2. A function $f \in \Sigma$ of the form (6) is said to be in the class $\Sigma_{\lambda}^{\mu}(\rho, \alpha, \beta, A, B)$ if it satisfies the subordination relation (19) above.

\section{Characterization and Other Related Properties}

In this section, we begin by proving a characterization property which provides a necessary and sufficient condition for a function $f \in \Sigma$ of the form (6) to belong to the class $\Sigma_{\lambda}^{\mu}(\rho, A, B)$ of meromorphically analytic functions.

Theorem 3. The function $f \in \Sigma$ is said to be a member of the class $\Sigma_{\lambda}^{\mu}(\rho, A, B)$ if and only if it satisfies

$$
\begin{aligned}
\sum_{n=1}^{\infty}\left(\frac{\lambda}{n+\lambda+1}\right)^{\mu}\left(\rho\left|b_{n}\right|(1+B)\right. & \\
& \left.-\left|c_{n}\right|(\rho(1+B)+A-B)\right)\left|a_{n}\right| \leq A-B .
\end{aligned}
$$

The equality is attained for the function $f_{n}(z)$ given by

$$
\begin{aligned}
f_{n}(z)= & \frac{1}{z} \\
& +\sum_{n=1}^{\infty} \frac{(A-B)(n+\lambda+1)^{\mu}}{\lambda^{\mu}\left(\rho\left|b_{n}\right|(1+B)-\left|c_{n}\right|(\rho(1+B)+A-B)\right)} z^{n} .
\end{aligned}
$$

Proof. Let $f$ of the form (6) belong to the class $\Sigma_{\lambda}^{\mu}(\rho, A, B)$. Then, in view of (12), we find that

$$
\begin{gathered}
\mid \rho \sum_{n=1}^{\infty}\left(\frac{\lambda}{n+\lambda+1}\right)^{\mu} a_{n}\left(b_{n}-c_{n}\right) z^{n+1} \\
\times((A-B) \\
-\sum_{n=1}^{\infty}\left(\frac{\lambda}{n+\lambda+1}\right)^{\mu}
\end{gathered}
$$

$$
\begin{aligned}
& \left.\times\left(\rho B b_{n}+c_{n}\{(A-B)-\rho B\}\right) a_{n} z^{n+1}\right)^{-1} \mid \\
& \leq \rho \sum_{n=1}^{\infty}\left(\frac{\lambda}{n+\lambda+1}\right)^{\mu} a_{n}\left(b_{n}-c_{n}\right)\left|z^{n+1}\right| \\
& \times((A-B) \\
& \quad-\sum_{n=1}^{\infty}\left(\frac{\lambda}{n+\lambda+1}\right)^{\mu} \\
& \left.\quad \times\left(\rho B b_{n}+c_{n}\{(A-B)-\rho B\}\right) a_{n}\left|z^{n+1}\right|\right)^{-1}
\end{aligned}
$$

$\leq 1$

Putting $|z|=r(0 \leq r<1)$ and noting the fact that the denominator in the above inequality remains positive by virtue of the constraints stated in (13) for all $r \in[0,1)$, we easily arrive at the desired inequality (20) by letting $z \rightarrow 1$.

Conversely, if we assume that the inequality (20) holds true in the simplified form (22), it can readily be shown that

$$
\left|\frac{\rho\{((f * g)(z))-((f * h)(z))\}}{\rho B((f * g)(z))+\{\rho(A-B)-\rho B\}((f * h)(z))}\right|<1,
$$

$$
\left(z \in U^{*}\right) \text {, }
$$

which is equivalent to our condition of theorem, so that $f \in$ $\Sigma_{\lambda}^{\mu}(\rho, A, B)$, hence the theorem.

Theorem 3 immediately yields the following result.

Corollary 4. If the function $f \in \sum$ belongs to the class $\Sigma_{\lambda}^{\mu}(\rho, A, B)$, then

$$
\left|a_{n}\right| \leq \frac{(A-B)(n+\lambda+1)^{\mu}}{\lambda^{\mu}\left(\rho\left|b_{n}\right|(1+B)-\left|c_{n}\right|(\rho(1+B)+A-B)\right)}, \quad n \geq 1,
$$

where the equality holds true for the functions $f_{n}(z)$ given by (21).

We now state the following growth and distortion properties for the class $\Sigma_{\lambda}^{\mu}(\rho, A, B)$.

Theorem 5. If the function $f$ defined by (6) is in the class $\Sigma_{\lambda}^{\mu}(\rho, A, B)$, then, for $0<|z|=r<1$, one has

$$
\begin{aligned}
\frac{1}{r} & -\frac{(A-B)(2+\lambda)^{\mu}}{\lambda^{\mu}\left(\rho\left|b_{1}\right|(1+B)-\left|c_{1}\right|(\rho(1+B)+A-B)\right)} r \\
& \leq|f(z)| \\
& \leq \frac{1}{r}+\frac{(A-B)(2+\lambda)^{\mu}}{\lambda^{\mu}\left(\rho\left|b_{1}\right|(1+B)-\left|c_{1}\right|(\rho(1+B)+A-B)\right)} r,
\end{aligned}
$$




$$
\begin{aligned}
\frac{1}{r^{2}}- & \frac{(A-B)(2+\lambda)^{\mu}}{\lambda^{\mu}\left(\rho\left|b_{1}\right|(1+B)-\left|c_{1}\right|(\rho(1+B)+A-B)\right)} \\
& \leq\left|f^{\prime}(z)\right| \\
& \leq \frac{1}{r^{2}}+\frac{(A-B)(2+\lambda)^{\mu}}{\lambda^{\mu}\left(\rho\left|b_{1}\right|(1+B)-\left|c_{1}\right|(\rho(1+B)+A-B)\right)} .
\end{aligned}
$$

Proof. Since $f \in \Sigma_{\lambda}^{\mu}(\rho, A, B)$, Theorem 3 readily yields the inequality

$$
\sum_{n=1}^{\infty}\left|a_{n}\right| \leq \frac{(A-B)(2+\lambda)^{\mu}}{\lambda^{\mu}\left(\rho\left|b_{1}\right|(1+B)-\left|c_{1}\right|(\rho(1+B)+A-B)\right)} .
$$

Thus, for $0<|z|=r<1$ and utilizing (26), we have

$$
\begin{aligned}
|f(z)| & =\frac{1}{|z|}+\sum_{n=1}^{\infty}\left|a_{n}\right||z|^{n} \leq \frac{1}{r}+r \sum_{n=1}^{\infty}\left|a_{n}\right| \\
& \leq \frac{1}{r}+r \frac{(A-B)(2+\lambda)^{\mu}}{\lambda^{\mu}\left(\rho\left|b_{1}\right|(1+B)-\left|c_{1}\right|(\rho(1+B)+A-B)\right)}, \\
|f(z)| & =\frac{1}{|z|}-\sum_{n=1}^{\infty}\left|a_{n}\right||z|^{n} \geq \frac{1}{r}-r \sum_{n=1}^{\infty}\left|a_{n}\right| \\
& \geq \frac{1}{r}-r \frac{(A-B)(2+\lambda)^{\mu}}{\lambda^{\mu}\left(\rho\left|b_{1}\right|(1+B)-\left|c_{1}\right|(\rho(1+B)+A-B)\right)} .
\end{aligned}
$$

Also from Theorem 3, we get

$$
\sum_{n=1}^{\infty} n\left|a_{n}\right| \leq \frac{(A-B)(2+\lambda)^{\mu}}{\lambda^{\mu}\left(\rho\left|b_{1}\right|(1+B)-\left|c_{1}\right|(\rho(1+B)+A-B)\right)} .
$$

Hence

$$
\begin{aligned}
\left|f^{\prime}(z)\right| & =\frac{1}{|z|^{2}}+\sum_{n=1}^{\infty} n\left|a_{n}\right||z|^{n-1} \\
& \leq \frac{1}{r^{2}}+\sum_{n=1}^{\infty}\left|a_{n}\right| \\
& \leq \frac{1}{r^{2}}+\frac{(A-B)(2+\lambda)^{\mu}}{\lambda^{\mu}\left(\rho\left|b_{1}\right|(1+B)-\left|c_{1}\right|(\rho(1+B)+A-B)\right)}, \\
\left|f^{\prime}(z)\right| & =\frac{1}{|z|^{2}}-\sum_{n=1}^{\infty} n\left|a_{n}\right||z|^{n-1} \\
& \geq \frac{1}{r^{2}}-\sum_{n=1}^{\infty} n\left|a_{n}\right| \\
& \geq \frac{1}{r^{2}}-\frac{(A-B)(2+\lambda)^{\mu}}{\lambda^{\mu}\left(\rho\left|b_{1}\right|(1+B)-\left|c_{1}\right|(\rho(1+B)+A-B)\right)} .
\end{aligned}
$$

This completes the proof of Theorem 5.
We next determine the radii of meromorphic starlikeness and meromorphic convexity of the class $\Sigma_{\lambda}^{\mu}(\rho, A, B)$, which are given by Theorems 6 and 7 below.

Theorem 6. If the function $f$ defined by (6) is in the class $\Sigma_{\lambda}^{\mu}(\rho, A, B)$, then $f$ is meromorphic starlike of order $\delta$ in the disk $|z|<r_{1}$, where

$$
\begin{aligned}
r_{1}=\inf _{n \geq 1}\{ & (1-\delta) \\
& \times\left(\rho\left|b_{n}\right|(1+B)-\left|c_{n}\right|(\rho(1+B)+A-B)\right) \\
& \left.\times((A-B)(n+2-\delta))^{-1}\right\}^{1 /(n+1)} .
\end{aligned}
$$

The equality is attained for the function $f_{n}(z)$ given by (21).

Proof. It suffices to prove that

$$
\left|\frac{z(f(z))^{\prime}}{f(z)}+1\right| \leq 1-\delta
$$

For $|z|<r_{1}$, we have

$$
\begin{aligned}
& \left|\frac{z(f(z))^{\prime}}{f(z)}+1\right| \\
& \quad=\left|\frac{\sum_{n=1}^{\infty}(n+1)(\lambda /(n+1+\lambda))^{\mu} a_{n} z^{n}}{1 / z+\sum_{n=1}^{\infty}(\lambda /(n+1+\lambda))^{\mu} a_{n} z^{n}}\right| \\
& \quad=\left|\frac{\sum_{n=1}^{\infty}(n+1)(\lambda /(n+\lambda+1))^{\mu} a_{n} z^{n+1}}{1+\sum_{n=1}^{\infty}(\lambda /(n+\lambda+1))^{\mu} a_{n} z^{n+1}}\right| \\
& \quad \leq \frac{\sum_{n=1}^{\infty}(n+1)(\lambda /(n+\lambda+1))^{\mu}\left|a_{n}\right||z|^{n+1}}{1+\sum_{n=1}^{\infty}(\lambda /(n+\lambda+1))^{\mu}\left|a_{n}\right||z|^{n+1}} .
\end{aligned}
$$

Hence (32) holds true for

$$
\begin{aligned}
& \sum_{n=1}^{\infty}(n+1)\left(\frac{\lambda}{n+\lambda+1}\right)^{\mu}\left|a_{n}\right||z|^{n+1} \\
& \quad \leq(1-\delta)\left(1-\sum_{n=1}^{\infty}\left(\frac{\lambda}{n+\lambda+1}\right)^{\mu}\left|a_{n}\right||z|^{n+1}\right)
\end{aligned}
$$

or

$$
\frac{\sum_{n=1}^{\infty}(n+2-\delta)(\lambda /(n+\lambda+1))^{\mu}\left|a_{n}\right||z|^{n+1}}{(1-\delta)} \leq 1
$$

With the aid of (20) and (34), it is true to say that for fixed $n$

$$
\begin{aligned}
& \frac{(n+2-\delta)(\lambda /(n+1+\lambda))^{\mu}|z|^{n+1}}{(1-\delta)} \\
& \leq\left(\frac{\lambda}{n+\lambda+1}\right)^{\mu} \\
& \quad \times\left(\rho\left|b_{n}\right|(1+B)+\left|c_{n}\right|(\rho(1+B)+A-B)\right) \\
& \quad \times(A-B)^{-1}, \quad n \geq 1 .
\end{aligned}
$$


Solving (35) for $|z|$, we obtain

$$
\begin{aligned}
|z| \leq\{ & (1-\delta) \\
& \times\left(\rho\left|b_{n}\right|(1+B)+\left|c_{n}\right|(\rho(1+B)+A-B)\right) \\
& \left.\times((n+2-\delta)(A-B))^{-1}\right\}^{n+1} .
\end{aligned}
$$

This completes the proof of Theorem 6 .

Theorem 7. If the function $f$ defined by (6) is in the class $\Sigma_{\lambda}^{\mu}(\rho, A, B)$, then $f$ is meromorphic convex of order $\delta$ in the disk $|z|<r_{2}$, where

$$
\begin{aligned}
r_{2}=\inf _{n \geq 1}\{ & (1-\delta) \\
& \times\left(\rho\left|b_{n}\right|(1+B)-\left|c_{n}\right|(\rho(1+B)+A-B)\right) \\
& \left.\times(n(n+2-\delta)(A-B))^{-1}\right\}^{1 /(n+1)} .
\end{aligned}
$$

The equality is attained for the function $f_{n}(z)$ given by (21).

Proof. By using the same technique employed in the proof of Theorem 6, we can show that

$$
\left|\frac{z(f(z))^{\prime \prime}}{(f(z))^{\prime}}+2\right| \leq 1-\delta .
$$

For $|z|<r_{1}$ and with the aid of Theorem 3, we have the assertion of Theorem 7 .

\section{Applications Involving Generalized Hypergeometric Functions}

Theorem 8. The function $f \in \Sigma$ is said to be a member of the class $\Sigma_{\lambda}^{\mu}(\rho, \alpha, \beta, A, B)$ if and only if it satisfies

$$
\begin{gathered}
\sum_{n=1}^{\infty}\left(\rho\left|b_{n}\right|(1+B)-\left|c_{n}\right|(\rho(1+B)+A-B)\right) \\
\times \frac{(\alpha)_{n+1}}{(\beta)_{n+1}}\left(\frac{\lambda}{n+\lambda+1}\right)^{\mu}\left|a_{n}\right| \leq A-B .
\end{gathered}
$$

The equality is attained for the function $f_{n}(z)$ given by

$$
\begin{aligned}
& f_{n}(z) \\
& =\frac{1}{z} \\
& \quad+\sum_{n=1}^{\infty} \frac{(A-B)(n+\lambda+1)^{\mu}}{\lambda^{\mu}\left(\rho\left|b_{n}\right|(1+B)-\left|c_{n}\right|(\rho(1+B)+A-B)\right)} z^{n}, \\
& \quad n \geq 1 .
\end{aligned}
$$

Proof. By using the same technique employed in the proof of Theorem 3 along with Definition 2, we can prove Theorem 8 .
The following consequences of Theorem 8 can be deduced by applying (39) and (40) along with Definition 2.

Corollary 9. If the function $f \in \Sigma$ belongs to the class $\Sigma_{\lambda}^{\mu}(\rho, \alpha, \beta, A, B)$, then

$$
\begin{array}{r}
\left|a_{n}\right| \\
\leq \frac{(A-B)(n+\lambda+1)^{\mu}(\beta)_{n+1}}{\lambda^{\mu}\left(\rho\left|b_{n}\right|(1+B)-\left|c_{n}\right|(\rho(1+B)+A-B)\right)(\alpha)_{n+1}}, \\
n \geq 1,
\end{array}
$$

where the equality holds true for the functions $f_{n}(z)$ given by (40).

Corollary 10. If the function $f$ defined by (6) is in the class $\Sigma_{\lambda}^{\mu}(\rho, \alpha, \beta, A, B)$, then $f$ is meromorphic starlike of order $\delta$ in the disk $|z|<r_{3}$, where

$$
\begin{aligned}
r_{3}=\inf _{n \geq 1}\{ & (1-\delta) \\
& \times\left(\rho\left|b_{n}\right|(1+B)-\left|c_{n}\right|(\rho(1+B)+A-B)\right) \\
& \left.\times((A-B)(n+2-\delta))^{-1}\right\}^{1 /(n+1)} .
\end{aligned}
$$

The equality is attained for the function $f_{n}(z)$ given by (40).

Corollary 11. If the function $f$ defined by (6) is in the class $\Sigma_{\lambda}^{\mu}(\rho, \alpha, \beta, A, B)$, then $f$ is meromorphic convex of order $\delta$ in the disk $|z|<r_{4}$, where

$$
\begin{aligned}
r_{4}=\inf _{n \geq 1}\{ & (1-\delta) \\
& \times\left(\rho\left|b_{n}\right|(1+B)-\left|c_{n}\right|(\rho(1+B)+A-B)\right) \\
& \left.\times(n(n+2-\delta)(A-B))^{-1}\right\}^{1 /(n+1)} .
\end{aligned}
$$

The equality is attained for the function $f_{n}(z)$ given by (40).

\section{Conflict of Interests}

The authors declare that there is no conflict of interests regarding the publication of this paper.

\section{Authors' Contribution}

All authors read and approved the final paper.

\section{Acknowledgment}

The work here was fully supported by FRGSTOPDOWN/ 2013/ST06/UKM/01/1.

\section{References}

[1] S. G. Krantz, "Meromorphic functions and singularities at infinity," in Handbook of Complex Variables, pp. 63-68, Birkhäauser, Boston, Mass, USA, 1999. 
[2] A. W. Goodman, "Functions typically-real and meromorphic in the unit circle," Transactions of the American Mathematical Society, vol. 81, pp. 92-105, 1956.

[3] N. E. Cho, O. S. Kwon, and H. M. Srivastava, "Inclusion and argument properties for certain subclasses of meromorphic functions associated with a family of multiplier transformations," Journal of Mathematical Analysis and Applications, vol. 300, no. 2, pp. 505-520, 2004.

[4] F. Ghanim and M. Darus, "Some properties on a certain class of meromorphic functions related to Cho-Kwon-Srivastava operator," Asian-European Journal of Mathematics, vol. 5, no. 4, Article ID 1250052, pp. 1-9, 2012.

[5] N. E. Cho and I. H. Kim, "Inclusion properties of certain classes of meromorphic functions associated with the generalized hypergeometric function," Applied Mathematics and Computation, vol. 187, no. 1, pp. 115-121, 2007.

[6] J. Dziok and H. M. Srivastava, "Some subclasses of analytic functions with fixed argument of coefficients associated with the generalized hypergeometric function," Advanced Studies in Contemporary Mathematics (Kyungshang), vol. 5, no. 2, pp. 115$125,2002$.

[7] J. Dziok and H. M. Srivastava, "Certain subclasses of analytic functions associated with the generalized hypergeometric function," Integral Transforms and Special Functions, vol. 14, no. 1, pp. 7-18, 2003.

[8] F. Ghanim, " A study of a certain subclass of Hurwitz-LerchZeta function related to a linear operator," Abstract and Applied Analysis, vol. 2013, Article ID 763756, 7 pages, 2013.

[9] F. Ghanim and M. Darus, "A new class of meromorphically analytic functions with applications to the generalized hypergeometric functions," Abstract and Applied Analysis, vol. 2011, Article ID 159405, 10 pages, 2011.

[10] F. Ghanim, M. Darus, and Z.-G. Wang, "Some properties of certain subclasses of meromorphically functions related to chokwon-srivastava operator," Information Journal, vol. 16, no. 9, pp. 6855-6866, 2013.

[11] J. Liu and H. M. Srivastava, "A linear operator and associated families of meromorphically multivalent functions," Journal of Mathematical Analysis and Applications, vol. 259, no. 2, pp. 566581, 2001.

[12] J. Liu and H. M. Srivastava, "Classes of meromorphically multivalent functions associated with the generalized hypergeometric function," Mathematical and Computer Modelling, vol. 39, no. 1, pp. 21-34, 2004. 


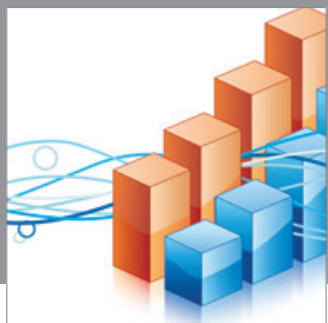

Advances in

Operations Research

mansans

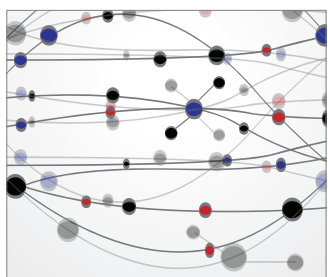

The Scientific World Journal
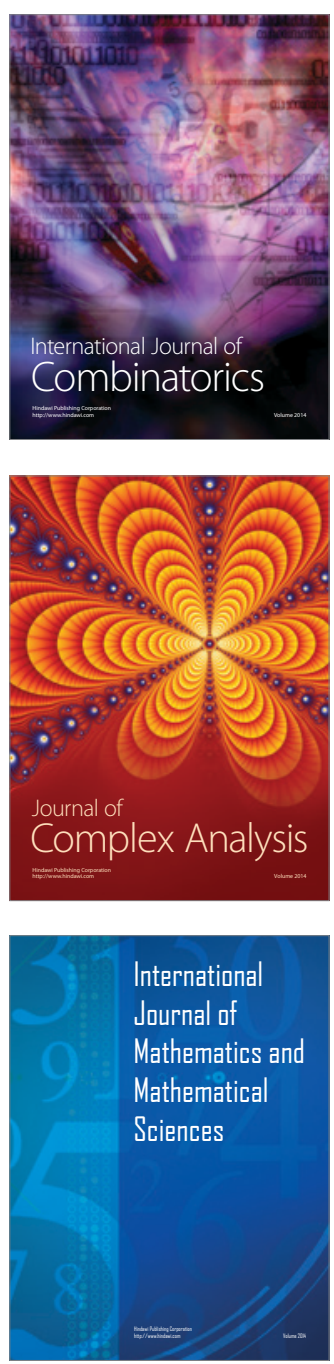
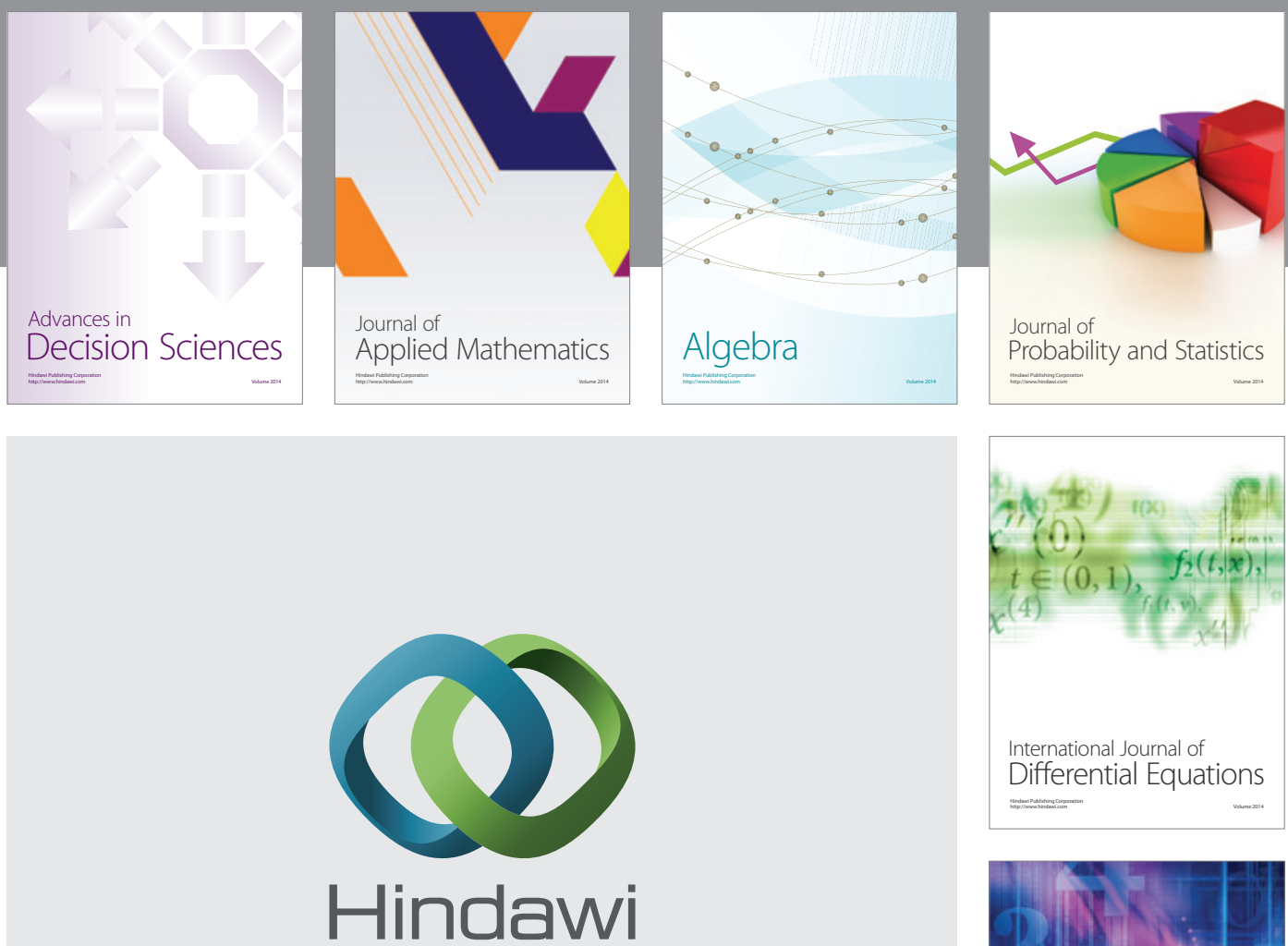

Submit your manuscripts at http://www.hindawi.com
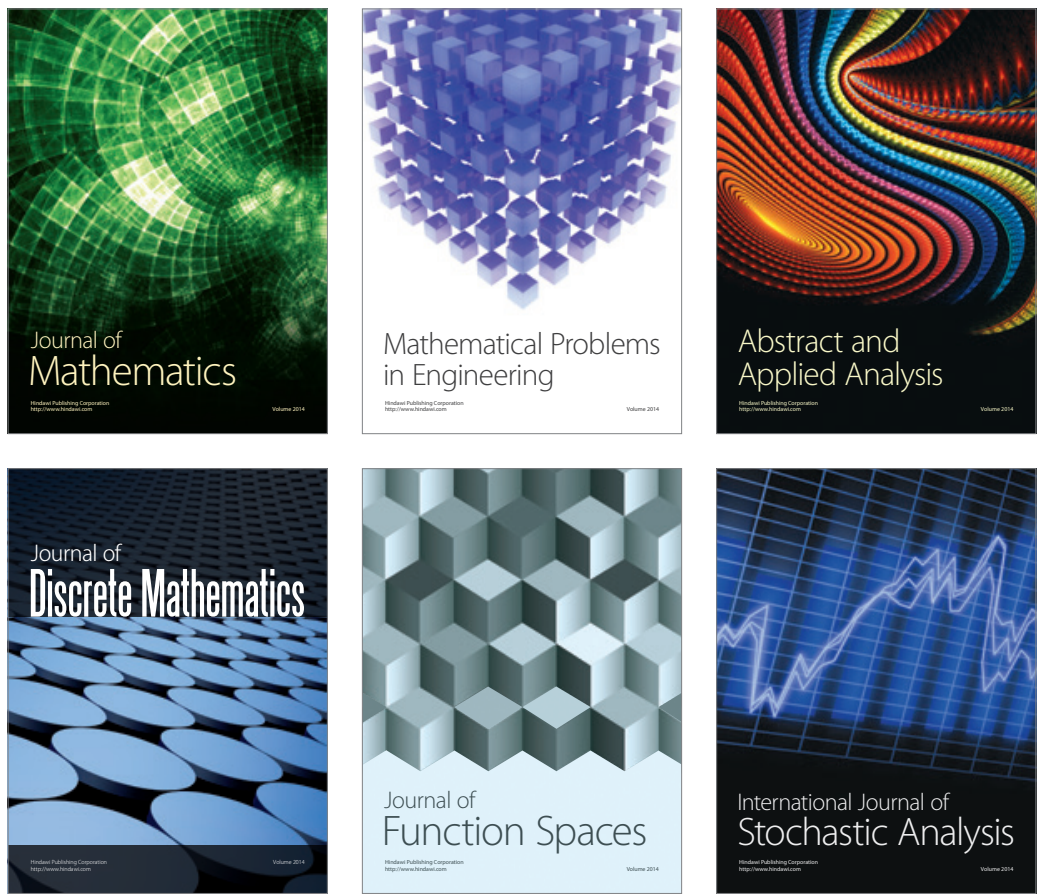

Journal of

Function Spaces

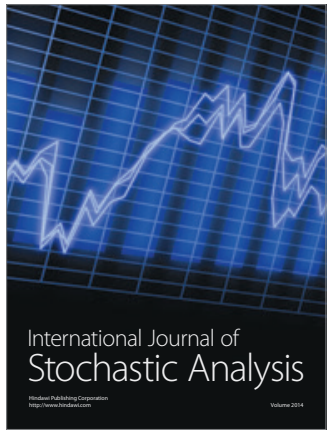

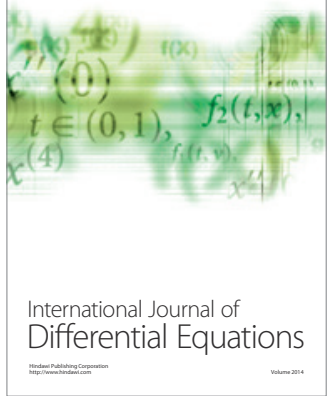
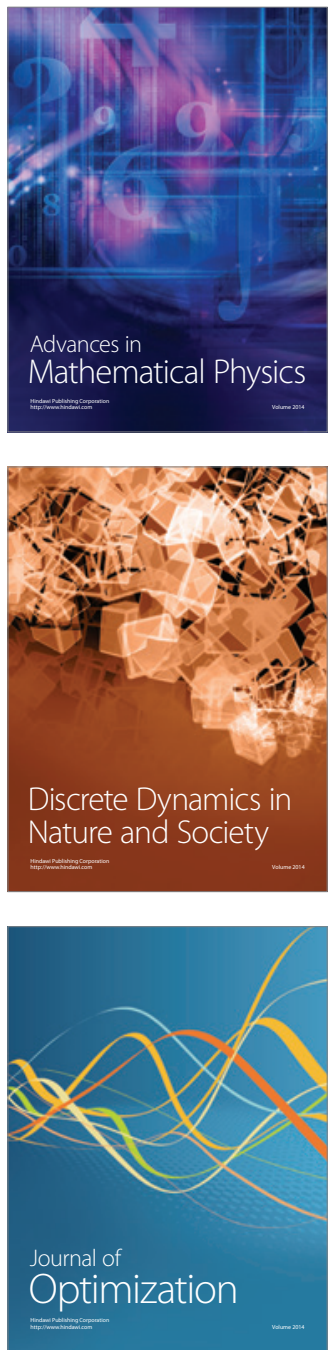